\title{
Spotlight
}

Korrespondenzadresse Prof. Dr. med. Claudia Pföhler

Universitätsklinikum des Saarlandes

Klinik für Dermatologie, Venerologie und Allergologie Kirrbergerstr., Geb. 18 66421 Homburg/Saar Claudia.Pfoehler@uniklinikumsaarland.de

\section{Infantile Hämangiome}

\section{Kurzkommentar zu Seite 406}

Im Übersichtsartikel von Marc Pleimes werden die Pathogenese, die Diagnostik und Therapie infantiler Hämangiome eingehend beleuchtet. Der Autor grenzt v.a. andere vaskuläre Anomalien wie das rapidly involuting congenital hemangiome (RICH), das non involuting congenital hemangioma (NICH) sowie andere Entitäten wie das Granuloma pygenicum, das Tufted angioma, das kaposiforme Hämangioendotheliom und vaskuläre Malformationen ab und zeigt deren Unterschiede zu infantilen Hämangiomen auf. Pleimes erläutert eindrücklich, wann es insbesondere aufgrund der Lokalisation der infantilen Hämangiome zu Komplikationen kommen kann und wann deswegen eine rasche Therapie erforderlich ist. In Bezug auf Kontrolle und Therapie wird verdeutlicht, dass insbesondere am Anfang eine engmaschige Kontrolle des Befundes notwendig ist. Als Faustregel gelten hierbei Alter des Kindes in Monaten $=$ Kontrollabstände in Wochen. Im abschließenden Teil zur Therapie infantiler Angiome geht der Autor ausführlich und kritisch würdigend auf lokalen Therapieverfahren ein und zeigt auf, wann eine systemische Therapie indiziert ist.
Late-onset-Neurofibromatose bei einem 70-jährigen Patienten mit Lungentuberkulose

\section{$\nabla$}

\section{Kurzkommentar zu Seite 421}

In einem spannenden Fallbericht wird ein 70-jähriger Patient vorgestellt, bei dem es erst i. R. einer Lungentuberkulose im 6. Lebensjahrzehnt zum Auftreten von Neurofibromatose-typischen Hautveränderungen wie Neurofibromen und Café-aulait-Flecken gekommen war. Die Familienanamnese des Patienten in Bezug auf eine Neurofibromatose (NF) war negativ gewesen. Aufgrund der späten Manifestation und der fehlenden systemischen Beteiligung wurde bei dem betroffenen Patienten eine Neurofibromatose vom Typ VII nach Riccardi diagnostiziert. Diese Variante ist erst etwa zwanzig Mal in der Literatur beschrieben und zeichnet sich durch eine Erstmanifestation frühestens ab dem 3. Lebensjahrzehnt aus. In der Regel liegt eine reine Hautbeteiligung vor, wobei die betroffenen Patienten meist nur Neurofibrome der Haut aufweisen. Die Pathogenese der NF vom Typ VII ist noch weitgehend ungeklärt, es gibt aber Hinweise, dass eine Immunsuppression einen entscheidenden Realisationsfaktor darstellt. Im vorgestellten Fall erscheint die Lungentuberkulose eine entscheidende Rolle gespielt zu haben. Bei Auftreten von NF-typischen Hautveränderungen in höherem Lebensalter sollte daher an das Vorliegen einer NF Typ VII nach Riccardi gedacht und eine möglicherweise zugrunde liegende Immunsuppression ausgeschlossen werden. 